\title{
Sleeve resection for bronchial carcinoid tumour in two children under six years old
}

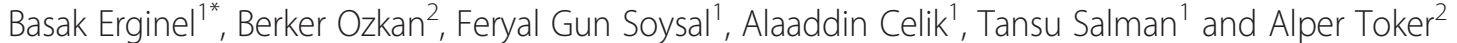

\begin{abstract}
Background: Paediatric tracheobronchial tumours are very rare, and pneumonectomy and lobectomy procedures are rarely indicated due to their surgical difficulties and high sequelae. Bronchoplastic techniques preserving lung parenchyma allow the resection and reconstruction of the main bronchi and carina.

Case Presentation: Here, we present a 6-year-old boy suffering from a carcinoid tumour of the right main bronchus which was successfully managed with a right upper sleeve lobectomy and a 4-year-old girl with an endobronchial carcinoid tumour narrowing the left main bronchus that received a sleeve resection of that bronchus.
\end{abstract}

Conclusion: Bronchoplastic techniques are widely used in adults, can be very successful in paediatric patients where the preservation of the lung parenchyma is more important.

Keywords: Bronchial carcinoid tumour, Sleeve resection, Paediatric

\section{Background}

The bronchial carcinoid (BC) tumour is a neuroendocrine tumour of the lung presenting in $1-2 \%$ of all lung neoplasia cases; however, $\mathrm{BC}$ tumours are very rare in the paediatric population. These tumours, which invade the airways causing a cough, haemoptysis and recurrent pulmonary infection, are categorised as typical carcinoid (TC) or atypical carcinoid (AC) according to their histopathologies [1]. Moreover, total surgical excision is important for a good prognosis. Recently, bronchoplastic techniques have been used to treat BC tumours in the airways of adults, and these techniques have also been suggested for paediatric patients, where the residual capacity after surgery is much more important [2]. However, the literature includes few reports of sleeve resections for $\mathrm{BC}$ tumours in the first decade of life [3].

\section{Case presentation}

Case 1

A 6-year-old boy presented at the paediatric intensive care unit of our hospital with acute respiratory

\footnotetext{
* Correspondence: basakerginel@hotmail.com

${ }^{1}$ Department of Pediatric Surgery, Istanbul Faculty of Medicine, Istanbul

University, Millet caddesi, Capa, 34093/Fatih, Istanbul, Turkey

Full list of author information is available at the end of the article
}

failure, and his chest $\mathrm{x}$-ray revealed a closed right hemithorax. A rigid bronchoscopic examination was done based on the suspicion of a foreign body, revealing an endobronchial mass on the right main bronchus. The histological examination of the biopsy was consistent with BC. Furthermore, his thoracic computed tomography $(\mathrm{CT})$ revealed a solid $18 \times 5 \mathrm{~mm}$ mass in the right main bronchus leading to the obstruction (Fig. 1).

To treat this patient, a right upper sleeve resection was performed under single-lung ventilation. The right main bronchus was dissected $0.5 \mathrm{~cm}$ distally from the carina, and an en bloc resection of the tumour was performed (Fig. 2). The surgical margins of the right main bronchus and intermediate bronchus were reported to be tumour-free in the evaluation of the frozen sections. The intermediate bronchus was then anastomosed to the right main bronchus at the level of the carina, with a continuous technique 4/0 PDS (Polydioxanone) suture in the membranous part and a separating technique in the cartilaginous part (Fig. 3).

Interlobar, hilar and mediastinal lymph node dissections were also conducted, and the pathological report confirmed T3N0M0 (stage IIB) typical BC with tumourfree margins. No short-term complications occurred; therefore, the patient was discharged on postoperative day 7 and has been under follow-up for 2 years. 


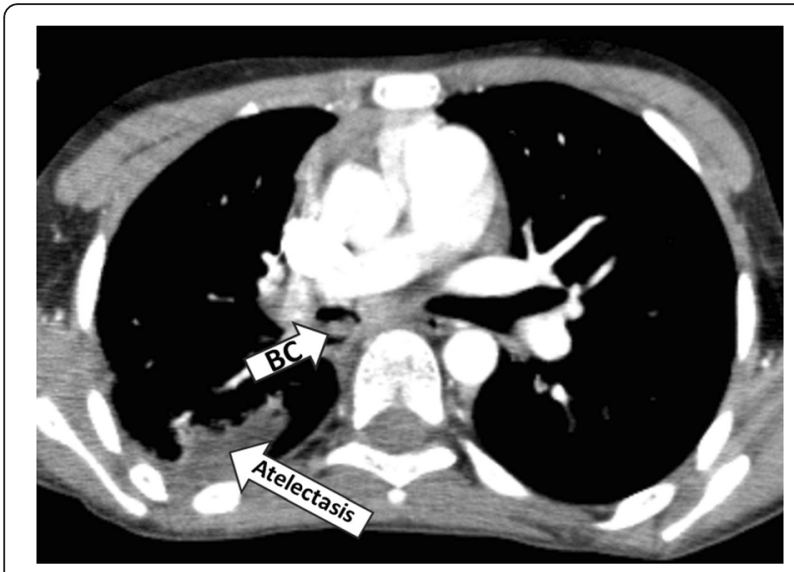

Fig. 1 Thoracic computed tomography of case 1, revealing a solid $18 \times 5 \mathrm{~mm}$ mass in the right main bronchus (BC) and post-obstructive atelectasis in the right upper lobe

\section{Case 2}

A 4-year-old girl was admitted to our department with complaints of coughing, wheezing and recurrent pneumonia, and her chest $\mathrm{x}$-ray revealed left atelectasis. Her thoracic CT revealed an endobronchial mass obstructing the left main bronchus, and rigid bronchoscopy showed an endobronchial mass narrowing the left main bronchus.

Under single-lung ventilation, this patient was treated with a sleeve resection of the left main bronchus and an end-to-end anastomosis with a continuous suture using 4/0 PDS in the membranous part and separate sutures for the cartilaginous part. A routine lymph node dissection was also performed, and the specimen was identified as T3N0M0 (stage IIB) typical BC with tumour-free margins. This patient was discharged on postoperative day 8 and has been under follow-up observation for 4 years without any recurrence.

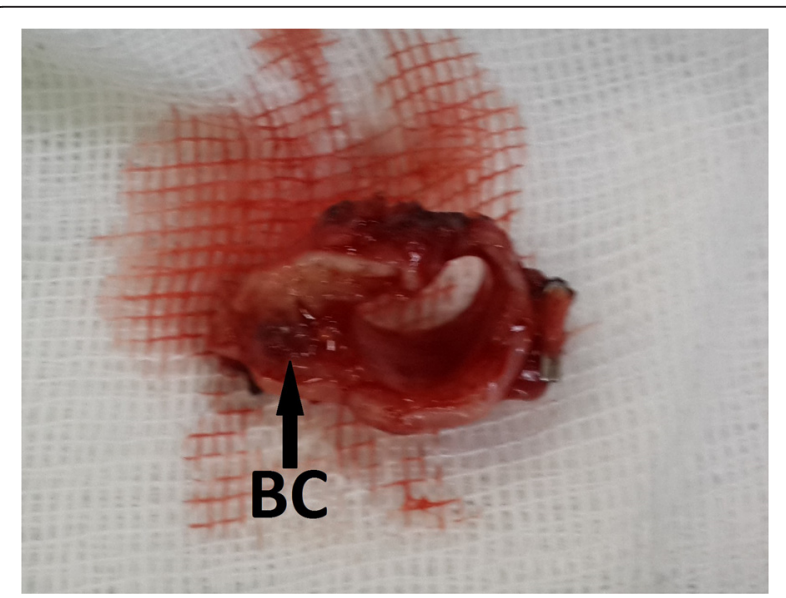

Fig. 2 Resected segment of the right main bronchus in case 1 ( $B C$ bronchial carcinoid tumour)

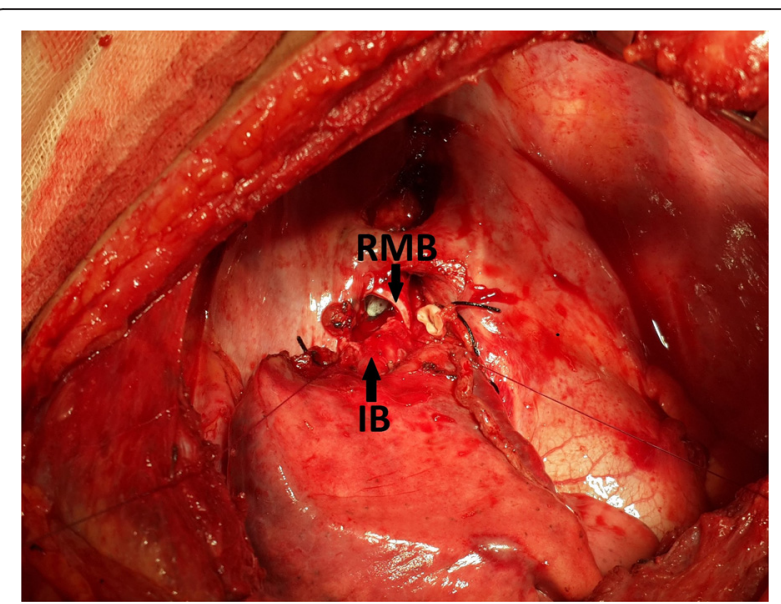

Fig. 3 Anastomosis of the intermediate bronchus to the right main bronchus at the level of the carina (RMB right main bronchus, IB intermediate bronchus)

\section{Discussion}

Primary pulmonary tumours in childhood are very rare, and of these rare tumours in the paediatric population, $\mathrm{BC}$ tumours (low-grade malignant neoplasms originating from neuroendocrine cells) are the most common [4]. Although pneumonectomies and lobectomies have typically been performed for bronchial tumours, the use of parenchyma-saving procedures, such as sleeve resections and bronchoplasties, has recently increased. It is extremely important to preserve the parenchyma to avoid the morbidity and sequelae that can occur throughout a patient's lifespan. Moreover, the aim of any surgical procedure is a resection with tumour-free margins while preserving the maximum amount of parenchyma. Only a few paediatric cases of $\mathrm{BC}$ treated with sleeve resections have been reported in the literature.

In the adult population, tracheobronchial sleeve resection is the treatment of choice for BC tumours for the best short- and long-term outcomes [5]. Gaissert et al. [2] proposed the procedure in the paediatric population and presented a series of 12 patients (aged 8-19 years old), including four with BC. Later, Toker et al. [3] reported two sleeve resections in patients under 10 years old for carcinoid tumours. Additionally, Rizzardi et al. [6] reported 15 children who underwent 10 parenchyma-saving procedures (five sleeve lobectomies, three sleeve resections of the main bronchus and two bronchoplasties).

Recently, there have been case reports of sleeve resections for other endobronchial tumours; for example, Pan et al. [7] reported the case of a 4-year-old boy with a myofibroblastic tumour who had a left inferior sleeve lobectomy. Additionally, Wildbrett et al. [8] reported the case of a 6-year-old boy with mucoepidermoid 
carcinoma who underwent a right upper sleeve resection, while de Agustín et al. [9] presented the case of a 5 -year-old girl who underwent sleeve resection for a mucoepidermoid carcinoma on the left main bronchus.

Sleeve bronchoplasty techniques are complex surgical procedures, especially in children, whose bronchial systems are much smaller and more delicate. In this case, we performed a bronchoscopy preoperatively and conducted perioperative frozen section studies and lymph node sampling in both of our cases (these are important to confirm tumour-free margins). Extracorporeal membrane oxygenation (ECMO) or similar treatments was not needed during or after the bronchial sleeve resection. Continuous suture 4/0 PDS was used in the membranous area, and a separating technique was used for the cartilaginous area in order to avoid anastomotic stricture during the follow-up period. For the postoperative follow-up of our patients, we used a CT scan, while the follow-up periods of our patients were 2 and 4 years without recurrence or any other complications.

As in our second case, an isolated sleeve of the main bronchus is a good choice for treatment in endobronchial main bronchi tumours in paediatric as well as adult patients in order to preserve the whole lung parenchyma [10]. Moreover, lymph node dissection for accurate staging is a major component of the surgical management of BCs, since these tumours have a greater than $10 \%$ risk of lymph node metastasis [11]. In both of our cases, we performed lymph node dissections.

Overall, $\mathrm{Yu}$ et al. [12] presented the largest comparison evaluating the efficacy of this treatment between paediatric and adult cases and found that sleeve resection also has an excellent prognosis in the paediatric population.

In the case of the failure of the anastomosis, the risk of a bronchopleural fistula and the aspiration of the empyema to the healthy lung should be kept in mind; therefore, in suspicious cases, the anastomosis should be rapidly checked via bronchoscopy. Previously, routine bronchoscopies were recommended for the control of each sleeve's bronchoplasty; however, they are not used now, although we do advise beginners in such bronchoplastic techniques to use them. Our second recommendation is to avoid excessive dissection for the prevention of major air leakage. Dissections under $5 \mathrm{~mm}$ are not recommended.

To the best of our knowledge, these are the first cases of $\mathrm{BC}$ tumours treated with sleeve resection in patients under the age of 5 years old. Since no series exists for this aspect of treatment, our case studies should encourage paediatric thoracic surgeons to utilise sleeve resection techniques in patients younger than 5 years old.

\section{Conclusions}

The sleeve lobectomy technique for $\mathrm{BC}$ tumors provides a better postoperative period, low postoperative morbidity and higher quality of life in the long term for a growing child. Bronchial reconstructive lung-sparing operations should be considered for appropriate bronchial tumours even in the first 5 years of life.

\section{Consent}

The patients' parents granted written informed consent for the publication of this manuscript and the accompanying images.

\section{Competing interests}

The authors declare that they have no competing interests.

Authors' contributions

All of the authors read and approved the final manuscript.

\section{Funding}

The authors received no financial support for the research or authorship of this article.

\section{Author details}

${ }^{1}$ Department of Pediatric Surgery, Istanbul Faculty of Medicine, Istanbul University, Millet caddesi, Capa, 34093/Fatih, Istanbul, Turkey. ${ }^{2}$ Department of Thoracic Surgery, Istanbul Faculty of Medicine, Istanbul University, Istanbul, Turkey.

Received: 24 June 2015 Accepted: 12 April 2016

Published online: 14 April 2016

\section{References}

1. Bagheri R, Mashhadi MT, Haghi SZ, Sadrizadh A, Rezaeetalab F. Tracheobronchopulmonary carcinoid tumors: analysis of 40 patients. Ann Thorac Cardiovasc Surg. 2011;17(1):7-12.

2. Gaissert HA, Mathisen DJ, Grillo HC, Vacanti JP, Wain JC. Tracheobronchial sleeve resection in children and adolescents. J Pediatr Surg. 1994;29(2):192-7.

3. Toker A, Bayrak Y, Dilege S, Kalayci G. Bronchial sleeve resections for carcinoid tumor in the first decade of life. Interact Cardiovasc Thorac Surg. 2004:3:280-2.

4. Rizzardi G, Marulli G, Calabrese F, et al. Bronchial carcinoid tumours in children: surgical treatment and outcome in a single institution. Eur J Pediatr Surg. 2009;19(4):228-31.

5. Erdoğan D, Serhan T, Berker Ö, et al. Bronșiyal karsinoid tümörlerde cerrahi tedavi: 11 yıllık deneyim. Türk Göğüs Kalp Damar Cerrahisi Dergisi. 2014;22(4):795-9.

6. Rizzardi G, Marulli G, Bortolotti L, Calabrese F, Sartori F, Rea F. Sleeve resections and bronchoplastic procedures in typical central carcinoid tumours. Thorac Cardiovasc Surg. 2008;56(1):42-5.

7. Pan W, Du L, Wu Y, Cai W. Successful sleeve lobectomy of inflammatory myofibroblastic tumor in a 4-year-old child. Ann Thorac Cardiovasc Surg. 2013;28.

8. Wildbrett P, Horras N, Lode H, Warzok R, Heidecke CD, Barthlen W. Mucoepidermoid carcinoma of the lung in a 6-year-old boy. Afr J Paediatr Surg. 2012;9(2):159-62.

9. de Agustín JC, Morcillo J, Millán A, Tuduri I, Granero R, Pérez BS. Bronchoplastic surgery: tumorectomy in principal left bronchial tube in a 5-month old child. Cir Pediatr. 2012;25(1):53-5.

10. Tang J, Cao M, Qian L, Fu Y, Tang J, Zhao X. The pure distal left main bronchial sleeve resection with total lung parenchymal preservation: report of two cases and literature review. J Thorac Dis. 2014;6(12):E294-8.

11. Nowak K, Karenovics W, Nicholson AG, Jordan S, Dusmet M. Pure bronchoplastic resections of the bronchus without pulmonary resection for endobronchial carcinoid tumours. Interact Cardiovasc Thorac Surg. 2013;17(2):291-4.

12. Yu Y, Song Z, Chen Z, Jian H, Lu S. Chinese pediatric and adolescent primary tracheobronchial tumors: a hospital-based study. Pediatr Surg Int. 2011;27(7):721-6. 